\title{
SCREENING OF WHEAT-THINOPYRUM BESSARABICUM ADDITION AND TRANSLOCATION LINES FOR DROUGHT TOLERANCE
}

\author{
SHAFQAT, N. ${ }^{*}{ }^{*}$ AHMED, H. ${ }^{2}-$ SHEHZAD, A. ${ }^{3}-$ CHAUdHRY, S. K. ${ }^{4}-$ SHAH, S. H. ${ }^{5}-$ ISLAM, M. $^{6}$ \\ - KHAN, W. ${ }^{7}-$ MASOOD, R. ${ }^{8}-$ KHAN, U. ${ }^{8}$ \\ ${ }^{1}$ Department of Agriculture, Hazara University, Mansehra, Pakistan \\ ${ }^{2}$ Islamia College, Peshawar, Pakistan
}

${ }^{3}$ National Institute for Genomics and Advanced Biotechnology (NIGAB), National Agricultural Research Centre (NARC), Islamabad, Pakistan

${ }^{4}$ Department of Botany, The University of Lahore, Sargodha Campus, Sargodha, Pakistan

${ }^{5}$ Department of Agricultural Sciences, Allama Iqbal Open University, Islamabad, Pakistan

${ }^{6}$ Department of Genetics, Hazara University, Mansehra, Pakistan

${ }^{7}$ Department of Environmental Sciences, Comsats Institute of Information Technology, Abbottabad Campus, Abbottabad, Pakistan

${ }^{8}$ Department of Botany, Hazara University, Mansehra, Pakistan

*Corresponding author

e-mail: noshinshafqat@gmail.com

(Received $2^{\text {nd }}$ Mar 2019; accepted $2^{\text {nd }}$ Jul 2019)

\begin{abstract}
Domestication of the crop plants has narrowed the genetic base of the crops and thereby mounting their exposure towards global climate change, disease and insect epidemics. Genetic variability of modern days crops must be broadened to make crop production more sustainable against various biotic and abiotic stresses which are posturing major threats to the world. It is therefore, crucial to identify novel additional sources of durable resistance. Wheat wild relatives have provided valuable sources of genetic diversity for various biotic, abiotic and quality components. In this respect wide hybridization has become a promising breeding method for introducing new variability in crop plants by using the crop wild relatives. In the present research work wheat-Th. Bessarabicum amphiploid, addition and translocation lines have been screened. These genetic stocks were exposed to 15\% polyethylene glycerol (PEG) stress, to find out the drought tolerant addition and translocation lines by studying different physiological parameters. The screening of drought stress revealed that addition lines $2 \mathrm{JJ}, 4 \mathrm{JJ}, 5 \mathrm{JJ}, 7 \mathrm{JJ}$ and translocation line $\operatorname{Tr}-7$ exhibited tolerance attitude towards water deficient conditions.
\end{abstract}

Keywords: chlorophyll contents, proline, superoxide dismutase, drought tolerance, wheat (Thinopyrum bessarabicum) addition and translocation lines

\section{Introduction}

The negative effect of non-living factors on the growth and development of living organism is termed as abiotic factors. Abiotic stress factors occurred naturally that may cause harm to the plants and animals in the effected area. Abiotic stress is the most destructive one concerning the growth and productivity of crops worldwide. It has also been found that abiotic factors are very harmful when they occur together in 
combinations (Milter, 2006). The most important abiotic stress factors include salinity, drought and water logging which cause harm to the plants.

Water deficit condition imposes severe influences on growth and development of plants by causing changes at the physiological, metabolic and molecular levels $(\mathrm{Zu}$ et al., 2017). Drought is the prolonged period without precipitation that is injurious to crops plants. It is the most important environmental factor that limits the plant growth and productivity than any other environmental stresses (Jones and Corlett, 1992). Approximately $32 \%$ of wheat growing culture faces different types of drought stress throughout the growing season in developing countries (Morris et al., 1991). About 15 million ha of cultivated land in Pakistan is affected by this environmental factor (Mujtaba and Alam, 2002). About one-third of the total annual cereal production is obtained from wheat alone. The response of plants for drought stress depends upon the severity, frequency, duration of exposure and the plant growth stage (Bray, 1993).

Germination, tillering and the reproductive stages are considered to be the most sensitive to drought in wheat (Passioura, 2007). The best way to encounter this problem is to develop high yielding drought tolerant crop varieties. Genetic diversity of cultivated plants has become drastically narrowed due to selection pressure in breeding programs thereby minimizing tolerance to environmental stresses (Nevo, 2004). Global warming followed by rising drought and salinity has threatened the world's food security. One of the economical and sustainable solutions for increasing crop stability and productivity is by making improvement in crops genetic structure for higher tolerance against abiotic stresses (Blum, 1998; Ashraf et al., 2009). This can be achieved by the utilization of several methods; one of the method is by identifying agronomically important novel genes and QTLs from the wild genotypes to improve the cultivars (Xie and Nevo, 2008).

The crop wild relatives that adapt to drought stress environment are expected to have genes or alleles for drought and salt tolerance. These drought tolerant genes and/or QTLs could be introgressed or cloned to increase crop tolerance (Araus et al., 2003). Addition of barley chromosome segment into wheat has shown tolerance to drought (Hoffmann et al., 2011). Th. bessarabicum, a wheat wild relative grows on the sea shore of the Crimea (Gorham et al., 1985) are highly salt tolerant. Wild relatives that show salt tolerance also exhibit drought tolerance (Farooq and Azam, 2001). Soil water potential is decreased in drought stress which ultimately reduces plants osmotic potential for water absorption by congestion of soluble carbohydrates and proline and in other words osmotic regulation is performed (Martin et al., 1993). Consequently osmotic regulation will help to improve plant growth and development in water stress (Pessarkli, 1999).

Drought tolerance can be enhanced if selection is made on the basis of physiological mechanisms or characters conferring tolerance. Drought stress tolerance in crop plants is associated with various physiological traits such as proline accumulation for osmoregulation (Nayyar and Walia, 2003; Larkunthod et al., 2018) increased activity of antioxidant enzymes like superoxide dismutase (SOD), peroxidases (POX) to protect membrane integrity (Sairam and Saxena, 2000) and various yield components (Majid et al., 2007; Lonbani and Arzani, 2011). One of the ways to develop drought tolerant crop, these parameters can be used to screen the genetic stock to identify the new sources of tolerance for developing drought tolerant crop species/varieties. Drought tolerant selection at seedling stage is mostly carried out through Polyethylene glycol (PEG)treated hydroponic conditions which induce negative osmotic potential comparable with 
moisture deficit stress (Robin et al., 2015). The aim of present experiment was to find out the suitable drought tolerant wheat-Th. bessarabicum genetic stocks. The evaluation was made on the basis of physiological responses like accumulation of proline, superoxide dismutase enzymes activity and chlorophyll contents.

\section{Materials and methods}

\section{Plant material}

The germplasm consisted of wheat-Th. bessarabicum addition and translocation lines along with some other drought tolerant and susceptible cultivated wheat varieties (Bano et al., 2012) as a check. The material was obtained from Wheat Wide Crosses Program at NARC Islamabad. Pedigree of these genetic stocks is given in Table 1.

Table 1. Pedigree of wheat-Th. bessarabicum genetic stocks

\begin{tabular}{|c|c|}
\hline Genetic stock & Pedigree \\
\hline $\operatorname{Tr}-1$ & 6BS.6BL-6J (CS/Th.bess//CSph/3/4*Prinia \\
\hline $\operatorname{Tr}-2$ & 1DS.1JS \\
\hline $\operatorname{Tr}-3$ & 3JS.3BL, $(\mathrm{CS} / \mathrm{Th}$. bess $/ / \mathrm{CSph} / 3 / 3 *$ Prinia \\
\hline $\operatorname{Tr}-4$ & 1AS.1AL-1JL \\
\hline $\operatorname{Tr}-5$ & 7DS.7DL-4J, (CS/Th.bess//CSph/3/4*Prinia \\
\hline $\operatorname{Tr}-6$ & 6JS.7DL, (CS/Th.bess//CSph/3/4*Prinia \\
\hline $\operatorname{Tr}-7$ & 5JS.5DS.5DL \\
\hline Amphiploid & CS/Thinopyrum bessarabicum \\
\hline $1 \mathrm{JJ}$ & $\mathrm{CS}+1 \mathrm{JJ}(2 \mathrm{n}=6 \mathrm{x}=42+2=\mathrm{AABBDD}+1 \mathrm{JJ})$ \\
\hline $2 \mathrm{JJ}$ & $\mathrm{CS}+2 \mathrm{JJ}(2 \mathrm{n}=6 \mathrm{x}=42+2=\mathrm{AABBDD}+2 \mathrm{JJ})$ \\
\hline $3 \mathrm{JJ}$ & $\mathrm{CS}+3 \mathrm{JJ}(2 \mathrm{n}=6 \mathrm{x}=42+2=\mathrm{AABBDD}+3 \mathrm{JJ})$ \\
\hline $4 \mathrm{JJ}$ & $\mathrm{CS}+4 \mathrm{JJ}(2 \mathrm{n}=6 \mathrm{x}=42+2=\mathrm{AABBDD}+4 \mathrm{JJ})$ \\
\hline $5 \mathrm{JJ}$ & $\mathrm{CS}+5 \mathrm{JJ}(2 \mathrm{n}=6 \mathrm{x}=42+2=\mathrm{AABBDD}+5 \mathrm{JJ})$ \\
\hline $6 \mathrm{JJ}$ & $\mathrm{CS}+6 \mathrm{JJ}(2 \mathrm{n}=6 \mathrm{x}=42+2=\mathrm{AABBDD}+6 \mathrm{JJ})$ \\
\hline $7 \mathrm{JJ}$ & $\mathrm{CS}+7 \mathrm{JJ}(2 \mathrm{n}=6 \mathrm{x}=42+2=\mathrm{AABBDD}+7 \mathrm{JJ})$ \\
\hline $3 \mathrm{JJ} 42$ & $C S+\operatorname{Tr} 3 J(2 n=6 x=42=A A B B D D+\operatorname{Tr} 3 J)$ \\
\hline $3 \mathrm{JJ} 44$ & $\mathrm{CS}+\operatorname{Tr} 3 \mathrm{JJ}(2 \mathrm{n}=6 \mathrm{x}=42+2=\mathrm{AABBDD}+\operatorname{Tr} 3 \mathrm{JJ})$ \\
\hline Chakwal-97, Punjab-96 & Drought tolerant and susceptible wheat cultivars (Bano et al., 2012) \\
\hline
\end{tabular}

\section{Laboratory procedure}

The genetic stock was evaluated for several physiological and biochemical traits analysis in hydroponics with Polyethylene glycerol (PEG) induced drought under controlled environmental conditions of laboratory (Fig. 1). In the first phase, all the genetic stocks with two check cultivars were evaluated for drought analysis under two treatments namely control (Hoagland solution) and 15\% PEG. Fifteen seeds/genetic stock were germinated in petri plates. After confirming the chromosomes number, the germinated seedlings of 3-4 days were shifted in small pot trays containing 2:3 of compost and sand. After 10 days, seedlings were transferred to holes on the $5 \mathrm{~L}$ hydroponics boxes containing half strength Hoagland's nutrient solution (Hoagland and Arnon, 1950). The $\mathrm{pH}$ of nutrient solution was maintained between 5.5-6.0 with $1 \mathrm{~N} \mathrm{HCl}$ 
or $\mathrm{KOH}$. Air pumps were fitted for providing oxygen to the roots throughout the growing period. For inducing drought, polyethylene glycol (PEG 6000) was used. Young leaf samples of three plants from each genetic stock were collected for different biochemical analyses after seven days of $15 \%$ PEG for drought stress.

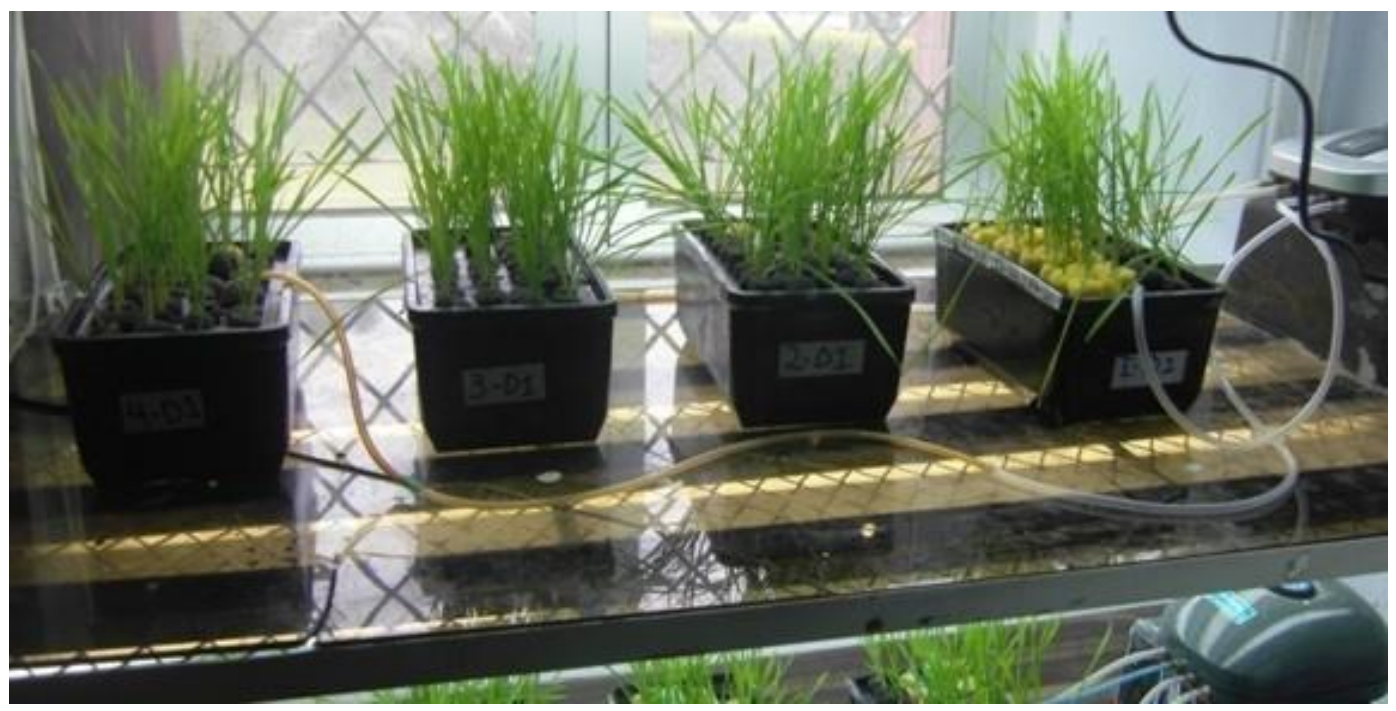

Figure 1. Hydroponic experiments for screening tolerance against drought in Thinopyrum bessarabicum genetic stock at $15 \%$ PEG treatment

\section{Proline contents determination}

Analysis of proline contents was done by the method of Bates et al. (1973). Fresh leaves of each sample weighing $0.25 \mathrm{~g}$ were homogenized in $10 \mathrm{~mL}$ of $3 \%$ aqueous sulfosalicylic acid and solution was filtered through Whatman 42 filter paper. $2 \mathrm{~mL}$ acid-ninhydrin and $2 \mathrm{~mL}$ of glacial acetic acid was added in filtrate and placed in a water bath for $1 \mathrm{~h}$ at $100{ }^{\circ} \mathrm{C}$. After that the mixture was extracted with $4 \mathrm{~mL}$ toluene and readings were taken at $520 \mathrm{~nm}$ wavelength using a spectrophotometer.

\section{Superoxide dismutase (SOD) determination}

The SOD activity was calculated by measuring the ability of the enzyme to inhibit phytochemical reduction of nitrobluetetrazolium (NBT) according to the method of Giannopolitis and Ries (1977). Fresh leaf sample $(0.25 \mathrm{~g})$ was homogenized with $1 \mathrm{~mL}$ of $100 \mathrm{mML}^{-1}$ sodium phosphate buffer containing $1 \%$ polyvinyl pyrollidone (PVP) in an ice bath. Then centrifugation was done at $10,000 \mathrm{rpm}$ for $15 \mathrm{~min}$ at $4{ }^{\circ} \mathrm{C}$. For SOD activity, the reaction mixture $(3 \mathrm{~mL})$ contained: K-Na-phosphate buffer $(60 \mathrm{mM}$, pH 7.8), methionine (13 $\left.\mathrm{mM} \mathrm{L}^{-1}\right)$, riboflavin $\left(12 \mathrm{mM} \mathrm{L}^{-1}\right)$, P-tetrazoleum blue $(80 \mu \mathrm{M})$, EDTA $(0.1 \mathrm{mM})$ and $100 \mu \mathrm{L}$ of enzyme extract. The reaction mixture was left for 10 min under $15 \mathrm{~W}$ fluorescent light. After $10 \mathrm{~min}$, light was switched off to stop the reaction. The absorbance of the mixture was measured by using spectrophotometer at A560 nm wavelength. For control the reaction mixture without the enzyme extract was utilized and a dark control mixture acted as a blank. One unit of SOD activity was taken as the quantity of enzyme that is able to inhibit tetrazoleum blue reduction by $50 \%$ as determined at $650 \mathrm{~nm}$ wavelength. SOD activity was expressed in arbitrary units per $\mathrm{mg}$ of fresh weight. 


\section{Chlorophyll contents determination}

Crude extract from each sample was taken and readings were measured at spectrophotometer (RS-110) at wavelength $645 \mathrm{~nm}$ and $663 \mathrm{~nm}$ immediately with care to minimize exposure to light. Chlorophyll contents were calculated according to Arnon (1949).

\section{Statistical analysis}

Completely Randomized Design (CRD) was followed with three replications. The data were analyzed by analysis of variance technique and comparison among treatment means was made by Least Significant Difference (LSD) test by using Statistix 8.1 computer software at $\mathrm{p} \leq 0.05$.

\section{Results}

\section{Proline contents}

Statistical analysis showed significant differences among wheat-Th. bessarabicum genetic stocks at $15 \%$ PEG stress at $\mathrm{p} \leq 0.05$ for proline contents (Table 2). In this experiment, free proline contents were increased in almost all the drought stressedseedlings of wheat genetic stock (Fig. 2). Maximum increase was observed in 3JJ (99\%) followed by $7 \mathrm{JJ}(96 \%)$ and 5JJ (93\%) disomic addition lines and in amphiploid (90\%) as compared to the non-stressed plants. These addition lines represented the increased proline contents as compared to CS parent and drought tolerant check cultivar Chakwal97, while addition lines $1 \mathrm{JJ}$ and $2 \mathrm{JJ}$ represented minimum increase in proline contents. All the translocation lines showed increase in proline contents than that of CS parent and tolerant check cultivar Chakwal-97. Translocation line Tr-6, Tr-7 and Tr-5 showed maximum increase of $97 \%, 95 \%$ and $94 \%$, respectively which is greater than that of the drought tolerant genotype Chakwal-97, CS parent and CS/Th. bessarabicum amphiploid.

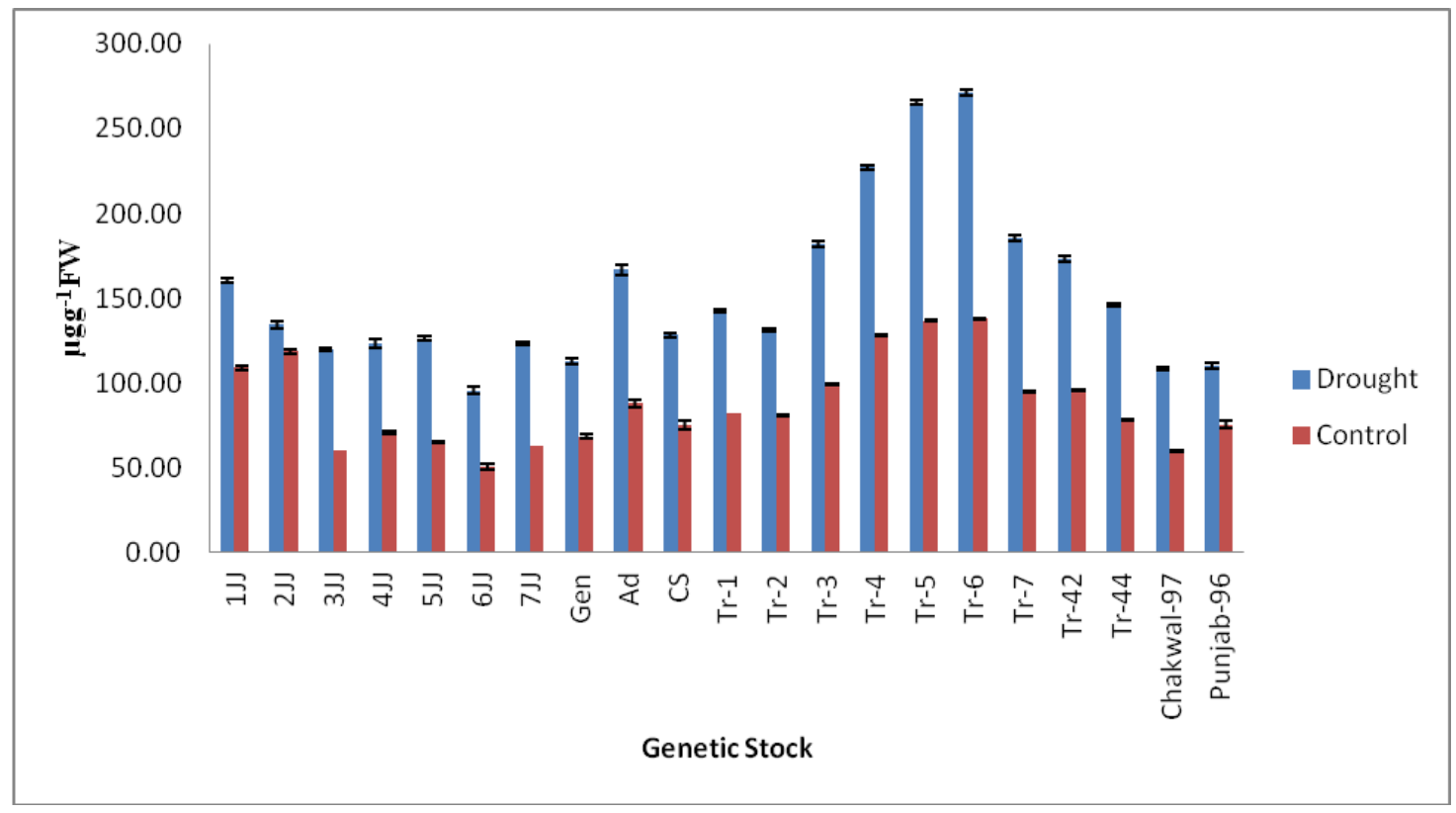

Figure 2. Proline contents in leaves at control and 15\% PEG treatments 
Table 2. Means for proline contents at 15\% PEG stress

\begin{tabular}{c|c|c|c}
\hline Genotypes & Control & Treatment & Mean \\
\hline 1JJ & $109.31 \mathrm{pq}$ & $160.34 \mathrm{~g}$ & $134.83 \mathrm{e}$ \\
2JJ & $118.72 \mathrm{o}$ & $134.42 \mathrm{ij}$ & $126.57 \mathrm{f}$ \\
3JJ & $60.38 \mathrm{yz}$ & $120.05 \mathrm{no}$ & $90.21 \mathrm{~m}$ \\
4JJ & $70.73 \mathrm{uv}$ & $123.35 \mathrm{mn}$ & $97.04 \mathrm{k}$ \\
5JJ & $65.23 \mathrm{wx}$ & $126.20 \mathrm{~lm}$ & $95.72 \mathrm{kl}$ \\
6JJ & $53.80 \mathrm{a}$ & $95.68 \mathrm{r}$ & $74.74 \mathrm{o}$ \\
$7 \mathrm{JJ}$ & $62.90 \mathrm{xy}$ & $123.10 \mathrm{mn}$ & $93.00 \mathrm{~lm}$ \\
Gen & $68.24 \mathrm{vw}$ & $112.91 \mathrm{p}$ & $90.58 \mathrm{~m}$ \\
Ad & $74.62 \mathrm{tu}$ & $166.71 \mathrm{f}$ & $120.66 \mathrm{~g}$ \\
CS & $75.12 \mathrm{t}$ & $128.36 \mathrm{kl}$ & $101.74 \mathrm{j}$ \\
Tr-1 & $82.11 \mathrm{~s}$ & $142.49 \mathrm{~h}$ & $112.30 \mathrm{~h}$ \\
Tr-2 & $80.74 \mathrm{~s}$ & $131.39 \mathrm{jk}$ & $106.07 \mathrm{i}$ \\
Tr-3 & $127.68 \mathrm{kl}$ & $227.44 \mathrm{c}$ & $177.56 \mathrm{c}$ \\
Tr-4 & $98.98 \mathrm{r}$ & $181.89 \mathrm{~d}$ & $140.44 \mathrm{~d}$ \\
$\operatorname{Tr}-5$ & $136.85 \mathrm{i}$ & $265.36 \mathrm{~b}$ & $201.11 \mathrm{~b}$ \\
$\operatorname{Tr}-6$ & $137.26 \mathrm{i}$ & $271.39 \mathrm{a}$ & $204.32 \mathrm{a}$ \\
Tr-7 & $95.48 \mathrm{r}$ & $185.34 \mathrm{~d}$ & $140.41 \mathrm{~d}$ \\
$\operatorname{Tr}-42$ & $78.38 \mathrm{st}$ & $173.10 \mathrm{e}$ & $125.74 \mathrm{f}$ \\
$\operatorname{Tr}-44$ & $58.99 \mathrm{yz}$ & $146.12 \mathrm{~h}$ & $102.55 \mathrm{j}$ \\
Chakwal-97 & $57.72 \mathrm{za}$ & $108.74 \mathrm{q}$ & $83.23 \mathrm{n}$ \\
Punjab-96 & $75.63 \mathrm{t}$ & $110.25 \mathrm{pq}$ & $92.94 \mathrm{~lm}$ \\
\hline
\end{tabular}

Inter critical value for comparison was 2.8 , while general critical value for comparison was 3.96

\section{Superoxide dismutase (SOD)}

Statistical analysis showed significant differences among wheat-Th. bessarabicum genetic stocks at $15 \%$ PEG stress at $\mathrm{p} \leq 0.05$ for superoxide dismutase activity (Table 3). The result showed SOD activity enhanced significantly in all the genotypes. The magnitude of the increment was different in various addition and translocation lines (Fig. 3). Under drought stress, maximum SOD activity was recorded in amphiploid (96\%), translocation line $\mathrm{Tr}-2$ (71\%), Tr-7 (75\%) and addition lines 1JJ (87\%), 2JJ (90\%), 5JJ (85\%), 7JJ (83\%) showed maximum SOD activity which was higher than that of the drought tolerant genotype Chakwal-97 (56\%). Other translocation and addition lines along with amphiploid showed high to medium increase in SOD activity.

\section{Chlorophyll A contents}

Statistical analysis showed significant differences among wheat-Th. bessarabicum genetic stocks at $15 \%$ PEG stress at $\mathrm{p} \leq 0.05$ for chlorophyll A contents (Table 4). Drought stress reduced significantly on leaf chlorophyll A contents of wheat genetic stock (Fig. 4). Comparison of wheat genetic stock revealed that addition lines 1JJ $(9.6 \%), 2 \mathrm{JJ}(17 \%)$ and $4 \mathrm{JJ}(12 \%)$ represented reduction in chlorophyll A contents as compared to control and drought tolerant check. Similarly translocation line Tr-7 (11\%), 
Tr-6(19\%) closely followed by Tr-3 (21\%) and Tr-4 (21\%) depicted minimum reduction in chlorophyll A contents. On the other hand, addition line $5 \mathrm{JJ}$ represented an increase in chlorophyll A contents.

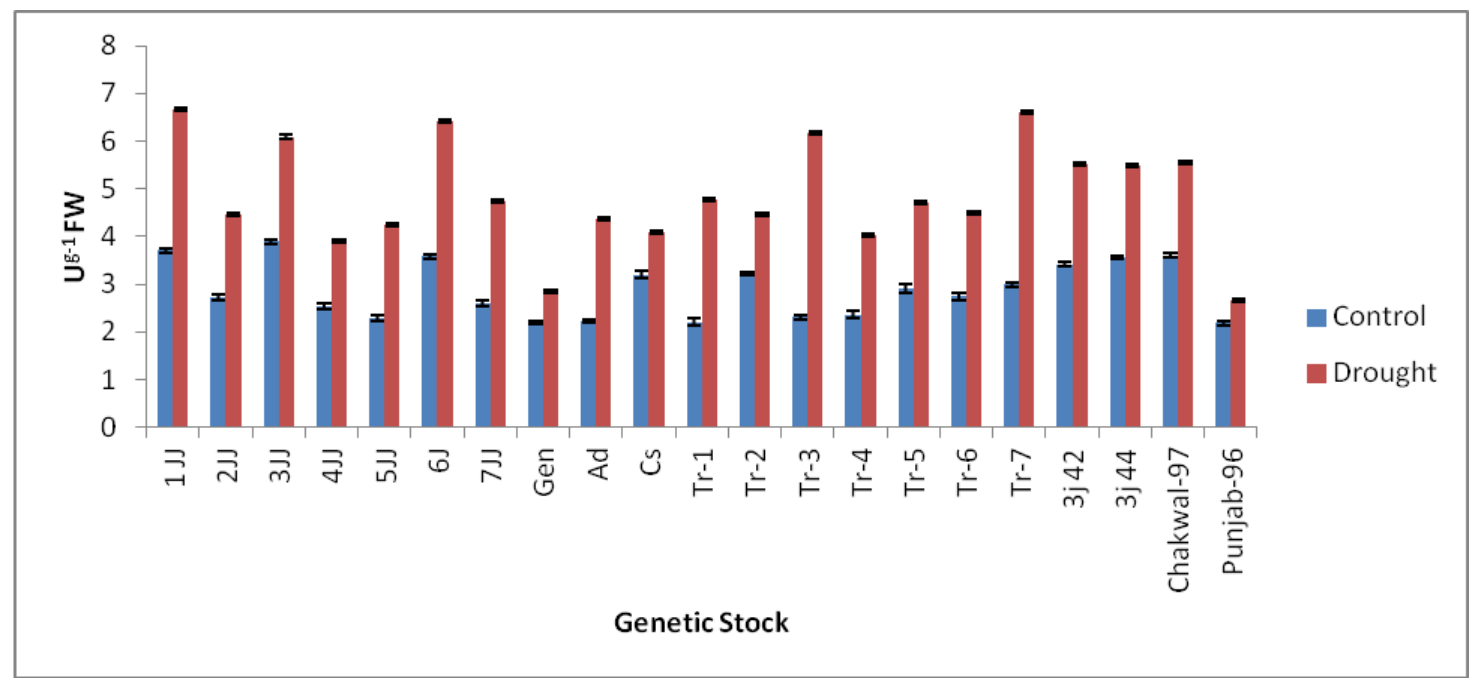

Figure 3. Superoxide dismutase (SOD) activity in leaves at control and 15\% PEG treatments

Table 3. Means for SOD contents at $15 \%$ PEG stress

\begin{tabular}{c|c|c|c}
\hline Genotypes & Control & Treatment & Mean \\
\hline 1JJ & $3.70 \mathrm{o}$ & $9.17 \mathrm{~b}$ & $6.43 \mathrm{a}$ \\
2JJ & $2.71 \mathrm{t}$ & $7.96 \mathrm{e}$ & $5.34 \mathrm{~g}$ \\
3JJ & $3.89 \mathrm{n}$ & $6.07 \mathrm{j}$ & $4.98 \mathrm{i}$ \\
4JJ & $2.53 \mathrm{u}$ & 5.171 & $3.85 \mathrm{~m}$ \\
5JJ & $2.28 \mathrm{vwx}$ & $9.61 \mathrm{a}$ & $5.95 \mathrm{~d}$ \\
6JJ & $3.57 \mathrm{p}$ & $8.90 \mathrm{c}$ & $6.23 \mathrm{c}$ \\
7JJ & $2.58 \mathrm{u}$ & $7.24 \mathrm{~g}$ & $4.91 \mathrm{j}$ \\
Gen & $2.19 \mathrm{xy}$ & $5.34 \mathrm{k}$ & $3.77 \mathrm{n}$ \\
Ad & $2.22 \mathrm{wxy}$ & $7.61 \mathrm{f}$ & $4.91 \mathrm{ij}$ \\
CS & $3.19 \mathrm{r}$ & $5.32 \mathrm{k}$ & 4.261 \\
Tr-1 & $2.20 \mathrm{wxy}$ & $9.25 \mathrm{~b}$ & $5.73 \mathrm{e}$ \\
Tr-2 & $3.20 \mathrm{r}$ & $2.93 \mathrm{~s}$ & $3.07 \mathrm{p}$ \\
Tr-3 & $2.30 \mathrm{vw}$ & $6.19 \mathrm{i}$ & $4.25 \mathrm{l}$ \\
Tr-4 & $2.35 \mathrm{v}$ & $6.52 \mathrm{~h}$ & $4.44 \mathrm{k}$ \\
Tr-5 & $2.92 \mathrm{~s}$ & $7.20 \mathrm{~g}$ & $5.06 \mathrm{~h}$ \\
Tr-6 & $2.74 \mathrm{t}$ & $4.49 \mathrm{~m}$ & $3.61 \mathrm{o}$ \\
Tr-7 & $2.99 \mathrm{~s}$ & $8.12 \mathrm{~d}$ & $5.55 \mathrm{f}$ \\
Tr-42 & $3.40 \mathrm{q}$ & $9.26 \mathrm{~b}$ & $6.33 \mathrm{~b}$ \\
Tr-44 & $3.55 \mathrm{p}$ & $7.99 \mathrm{e}$ & $5.77 \mathrm{e}$ \\
Chakwal-97 & $3.60 \mathrm{p}$ & $7.55 \mathrm{f}$ & $5.57 \mathrm{f}$ \\
Punjab-96 & $2.18 \mathrm{y}$ & $3.91 \mathrm{n}$ & $3.05 \mathrm{p}$ \\
\hline
\end{tabular}

Inter critical value for comparison was 0.09 , while general critical value for comparison was 0.06 


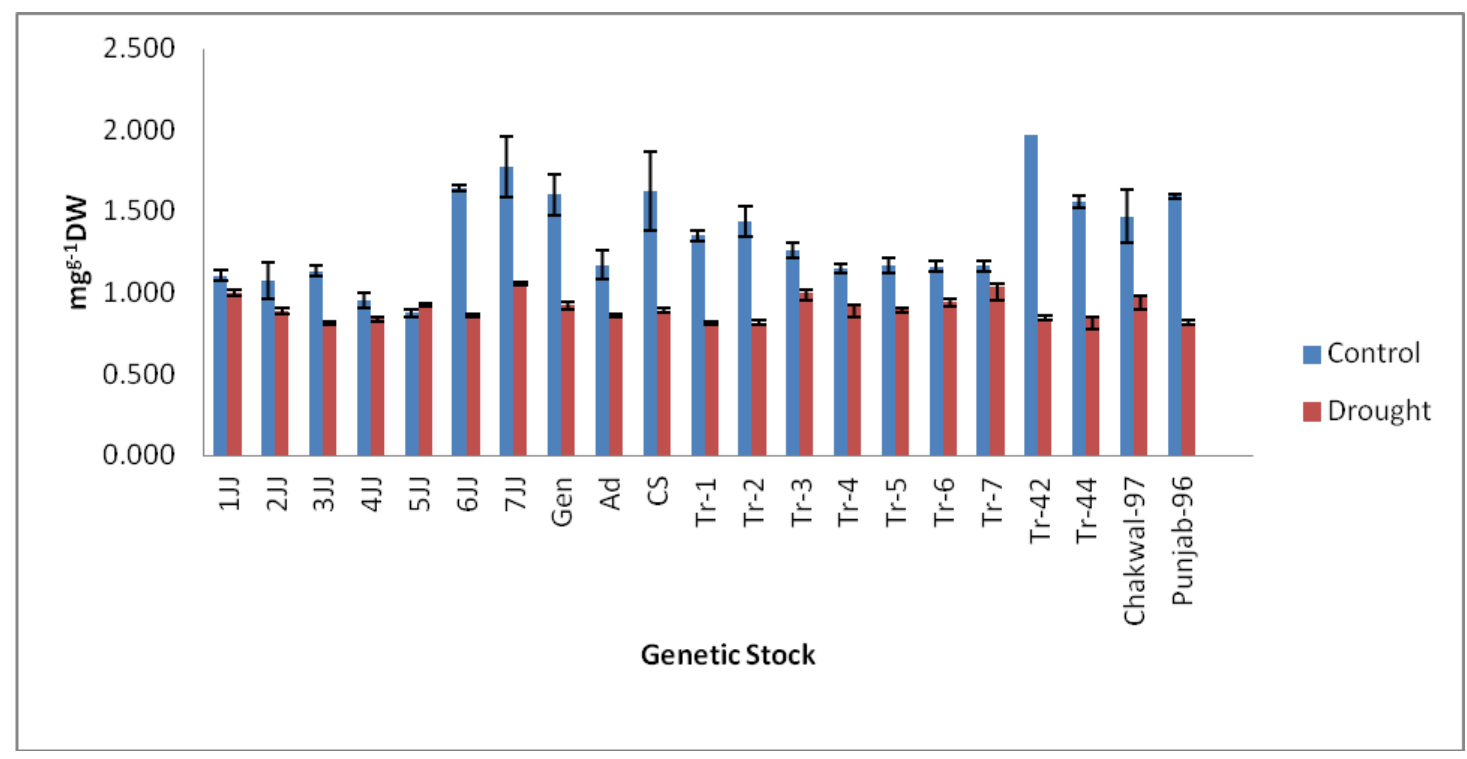

Figure 4. Chlorophyll A contents in leaves at control and $15 \%$ PEG treatments

Table 4. Means for chlorophyll A contents at 15\% PEG stress

\begin{tabular}{c|c|c|c}
\hline Genotypes & Control & Treatment & Mean \\
\hline 1JJ & $1.10 \mathrm{~h}-1$ & $0.99 \mathrm{i}-\mathrm{o}$ & $1.05 \mathrm{ef}$ \\
2JJ & $0.85 \mathrm{~m}-\mathrm{p}$ & $0.88 \mathrm{~m}-\mathrm{p}$ & $0.87 \mathrm{~h}$ \\
3JJ & $1.13 \mathrm{~h}-\mathrm{k}$ & $0.80 \mathrm{p}$ & $0.96 \mathrm{fgh}$ \\
4JJ & $0.95 \mathrm{k}-\mathrm{p}$ & $0.83 \mathrm{op}$ & $0.89 \mathrm{gh}$ \\
$5 \mathrm{JJ}$ & $0.8743 \mathrm{~m}-\mathrm{p}$ & $0.91 \mathrm{l}-\mathrm{p}$ & $0.89 \mathrm{gh}$ \\
6JJ & $1.6440 \mathrm{bc}$ & $0.85 \mathrm{~m}-\mathrm{p}$ & $1.24 \mathrm{bc}$ \\
$7 \mathrm{JJ}$ & $1.77 \mathrm{~b}$ & $1.04 \mathrm{i}-\mathrm{m}$ & $1.41 \mathrm{a}$ \\
Gen & $1.60 \mathrm{bcd}$ & $0.92 \mathrm{l}-\mathrm{p}$ & $1.26 \mathrm{~b}$ \\
Ad & $1.36 \mathrm{ef}$ & $0.85 \mathrm{~m}-\mathrm{p}$ & $1.11 \mathrm{cde}$ \\
CS & $1.62 \mathrm{bcd}$ & $0.88 \mathrm{~m}-\mathrm{p}$ & $1.25 \mathrm{~b}$ \\
Tr-1 & $1.35 \mathrm{fg}$ & $0.80 \mathrm{op}$ & $1.07 \mathrm{def}$ \\
Tr-2 & $1.43 \mathrm{def}$ & $0.81 \mathrm{op}$ & $1.12 \mathrm{~b}-\mathrm{e}$ \\
Tr-3 & $1.25 \mathrm{fgh}$ & $0.99 \mathrm{i}-\mathrm{p}$ & $1.12 \mathrm{~b}-\mathrm{e}$ \\
Tr-4 & $1.14 \mathrm{hij}$ & $0.91 \mathrm{l}-\mathrm{p}$ & $1.03 \mathrm{efg}$ \\
Tr-5 & $1.16 \mathrm{ghi}$ & $0.89 \mathrm{~m}-\mathrm{p}$ & $1.02 \mathrm{efg}$ \\
Tr-6 & $1.16 \mathrm{~g}-\mathrm{j}$ & $0.94 \mathrm{k}-\mathrm{p}$ & $1.05 \mathrm{ef}$ \\
Tr-7 & $1.16 \mathrm{ghi}$ & $1.03 \mathrm{i}-\mathrm{n}$ & $1.10 \mathrm{def}$ \\
Tr-42 & $1.97 \mathrm{a}$ & $0.84 \mathrm{nop}$ & $1.40 \mathrm{a}$ \\
Tr-44 & $1.55 \mathrm{cde}$ & $0.83 \mathrm{op}$ & $1.19 \mathrm{bcd}$ \\
Chakwal-97 & $2.00 \mathrm{a}$ & $0.96 \mathrm{j}-\mathrm{p}$ & $1.48 \mathrm{a}$ \\
Punjab-96 & $1.59 \mathrm{bcd}$ & $0.81 \mathrm{op}$ & $1.20 \mathrm{bcd}$ \\
\hline
\end{tabular}

Inter critical value for comparison was 0.19: while general critical value for comparison was 0.13 


\section{Chlorophyll B contents}

Statistical analysis showed significant differences among wheat-Th. bessarabicum genetic stocks at $15 \%$ PEG stress at $\mathrm{p} \leq 0.05$ for chlorophyll B contents (Table 5). Water deficit significantly reduced leaf chlorophyll B contents of all the wheat genetic stock (Fig. 5). Results revealed that all the wheat genetic stock addition lines 1JJ (24\%), 4JJ (38\%) 7JJ (39\%) and translocation line Tr-1 (18\%), Tr-9 (22\%) represented the lowest reduction in chlorophyll B contents as compared to control and drought tolerant genotype. While addition line 5JJ (61\%) and translocation line $\operatorname{Tr}-8(61 \%)$ depicted maximum decrease in chlorophyll $\mathrm{B}$ contents.

\section{Total chlorophyll contents}

Statistical analysis showed significant differences among wheat-Th. bessarabicum genetic stocks at $15 \%$ PEG stress at $\mathrm{p} \leq 0.05$ for total chlorophyll contents (Table 6). Results revealed that addition line 1JJ (14\%), 2JJ (10\%) and 4JJ (18\%) showed minimum reduction in total chlorophyll contents, while addition lines $5 \mathrm{JJ}(24 \%), 6 \mathrm{JJ}$ (24\%) and 7JJ (25\%) depicted moderate decrease in total chlorophyll contents (Fig. 6). Similarly translocation lines $\operatorname{Tr}-5$ and $\operatorname{Tr}-7$ represented lower reduction in total chlorophyll contents.

Table 5. Means for chlorophyll B contents at $15 \%$ PEG stress

\begin{tabular}{c|c|c|c}
\hline Genotypes & Control & Treatment & Mean \\
\hline $1 \mathrm{JJ}$ & $0.43 \mathrm{e}-\mathrm{l}$ & $0.32 \mathrm{i}-\mathrm{p}$ & $0.37 \mathrm{c}-\mathrm{f}$ \\
$2 \mathrm{JJ}$ & $0.45 \mathrm{~d}-\mathrm{k}$ & $0.29 \mathrm{k}-\mathrm{p}$ & $0.37 \mathrm{def}$ \\
$3 \mathrm{JJ}$ & $0.49 \mathrm{~d}-\mathrm{h}$ & $0.23 \mathrm{op}$ & $0.36 \mathrm{def}$ \\
$4 \mathrm{JJ}$ & $0.41 \mathrm{f}-\mathrm{n}$ & $0.25 \mathrm{~m}-\mathrm{p}$ & $0.33 \mathrm{f}$ \\
$5 \mathrm{JJ}$ & $0.68 \mathrm{ab}$ & $0.26 \mathrm{l-p}$ & $0.47 \mathrm{a}-\mathrm{d}$ \\
$6 \mathrm{JJ}$ & $0.49 \mathrm{~d}-\mathrm{i}$ & $0.25 \mathrm{~m}-\mathrm{p}$ & $0.37 \mathrm{def}$ \\
$7 \mathrm{JJ}$ & $0.60 \mathrm{a}-\mathrm{d}$ & $0.37 \mathrm{~g}-\mathrm{o}$ & $0.48 \mathrm{abc}$ \\
Gen & $0.53 \mathrm{~b}-\mathrm{g}$ & $0.24 \mathrm{op}$ & $0.38 \mathrm{~b}-\mathrm{f}$ \\
Ad & $0.48 \mathrm{~d}-\mathrm{j}$ & $0.24 \mathrm{nop}$ & $0.36 \mathrm{def}$ \\
CS & $0.41 \mathrm{f}-\mathrm{m}$ & $0.28 \mathrm{k}-\mathrm{p}$ & $0.35 \mathrm{ef}$ \\
Tr-1 & $0.50 \mathrm{c}-\mathrm{g}$ & $0.41 \mathrm{f}-\mathrm{m}$ & $0.45 \mathrm{a}-\mathrm{e}$ \\
Tr-2 & $0.48 \mathrm{~d}-\mathrm{i}$ & $0.20 \mathrm{p}$ & $0.34 \mathrm{ef}$ \\
Tr-3 & $0.54 \mathrm{~b}-\mathrm{f}$ & $0.27 \mathrm{l-p}$ & $0.41 \mathrm{a}-\mathrm{f}$ \\
Tr-4 & $0.69 \mathrm{ab}$ & $0.29 \mathrm{k}-\mathrm{p}$ & $0.49 \mathrm{abc}$ \\
Tr-5 & $0.54 \mathrm{~b}-\mathrm{f}$ & $0.28 \mathrm{l-p}$ & $0.41 \mathrm{a}-\mathrm{f}$ \\
Tr-6 & $0.72 \mathrm{a}$ & $0.32 \mathrm{j}-\mathrm{p}$ & $0.52 \mathrm{a}$ \\
Tr-7 & $0.66 \mathrm{abc}$ & $0.33 \mathrm{~h}-\mathrm{p}$ & $0.49 \mathrm{ab}$ \\
Tr-42 & $0.67 \mathrm{ab}$ & $0.26 \mathrm{~m}-\mathrm{p}$ & $0.46 \mathrm{a}-\mathrm{d}$ \\
Tr-44 & $0.41 \mathrm{f}-\mathrm{n}$ & $0.31 \mathrm{k}-\mathrm{p}$ & $0.36 \mathrm{def}$ \\
Chakwal-97 & $0.59 \mathrm{a}-\mathrm{e}$ & $0.25 \mathrm{~m}-\mathrm{p}$ & $0.42 \mathrm{a}-\mathrm{f}$ \\
Punjab-96 & $0.51 \mathrm{c}-\mathrm{g}$ & $0.261-\mathrm{p}$ & $0.38 \mathrm{~b}-\mathrm{f}$ \\
\hline
\end{tabular}

Inter critical value for comparison was 0.16 , while general critical value for comparison was 0.11 


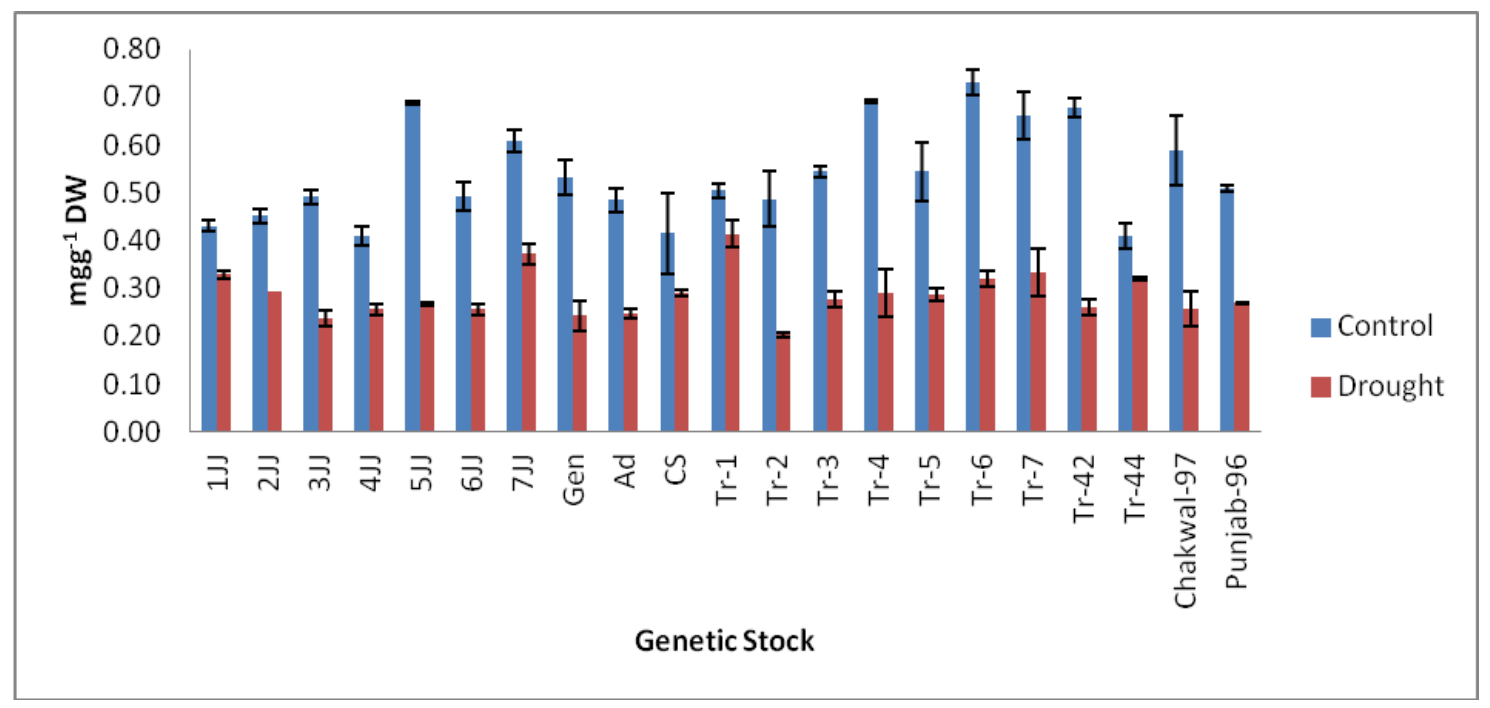

Figure 5. Chlorophyll B contents in leaves at control and 15\% PEG treatments

Table 6. Means for total chlorophyll contents at $15 \%$ PEG stress

\begin{tabular}{c|c|c|c}
\hline Genotypes & Control & Treatment & Mean \\
\hline 1JJ & $1.53 \mathrm{jk}$ & $0.62 \mathrm{mn}$ & $1.08 \mathrm{def}$ \\
2JJ & $1.31 \mathrm{k}$ & $0.47 \mathrm{l}-\mathrm{o}$ & $0.89 \mathrm{~g}$ \\
3JJ & $1.62 \mathrm{hij}$ & $0.33 \mathrm{o}$ & $0.98 \mathrm{fg}$ \\
4JJ & $1.36 \mathrm{k}$ & $0.39 \mathrm{no}$ & $0.87 \mathrm{~g}$ \\
5JJ & $1.56 \mathrm{ijk}$ & $0.48 \mathrm{l-o}$ & $1.02 \mathrm{efg}$ \\
6JJ & $2.13 \mathrm{~cd}$ & $0.40 \mathrm{mno}$ & $1.27 \mathrm{bc}$ \\
$7 \mathrm{JJ}$ & $2.38 \mathrm{bc}$ & 0.721 & $1.55 \mathrm{a}$ \\
Gen & $2.13 \mathrm{~cd}$ & $0.461-\mathrm{o}$ & $1.29 \mathrm{~b}$ \\
Ad & $1.85 \mathrm{e}-\mathrm{h}$ & $0.40 \mathrm{no}$ & $1.12 \mathrm{~b}-\mathrm{f}$ \\
CS & $2.04 \mathrm{def}$ & $0.471-\mathrm{o}$ & $1.26 \mathrm{bcd}$ \\
Tr-1 & $1.76 \mathrm{~g}-\mathrm{j}$ & $0.31 \mathrm{o}$ & $1.04 \mathrm{efg}$ \\
Tr-2 & $1.92 \mathrm{~d}-\mathrm{g}$ & $0.31 \mathrm{o}$ & $1.11 \mathrm{~b}-\mathrm{f}$ \\
Tr-3 & $1.80 \mathrm{f}-\mathrm{i}$ & $0.57 \mathrm{l}-\mathrm{o}$ & $1.18 \mathrm{~b}-\mathrm{e}$ \\
Tr-4 & $1.84 \mathrm{e}-\mathrm{h}$ & $0.50 \mathrm{l-o}$ & $1.17 \mathrm{~b}-\mathrm{e}$ \\
Tr-5 & $1.71 \mathrm{~g}-\mathrm{j}$ & $0.47 \mathrm{l-o}$ & $1.09 \mathrm{c}-\mathrm{f}$ \\
Tr-6 & $1.89 \mathrm{~d}-\mathrm{g}$ & $0.561-\mathrm{o}$ & $1.22 \mathrm{bcd}$ \\
Tr-7 & $1.82 \mathrm{f}-\mathrm{i}$ & $0.67 \mathrm{~lm}$ & $1.24 \mathrm{bcd}$ \\
Tr-42 & $2.65 \mathrm{a}$ & $0.40 \mathrm{no}$ & $1.52 \mathrm{a}$ \\
Tr-44 & $1.96 \mathrm{~d}-\mathrm{g}$ & $0.45 \mathrm{mno}$ & $1.20 \mathrm{~b}-\mathrm{e}$ \\
Chakwal-97 & $2.59 \mathrm{ab}$ & $0.52 \mathrm{l-o}$ & $1.55 \mathrm{a}$ \\
Punjab-96 & $2.10 \mathrm{de}$ & $0.38 \mathrm{no}$ & $1.24 \mathrm{bcd}$ \\
\hline
\end{tabular}

Inter critical value for comparison was 0.26 , while general critical value for comparison was 0.05 


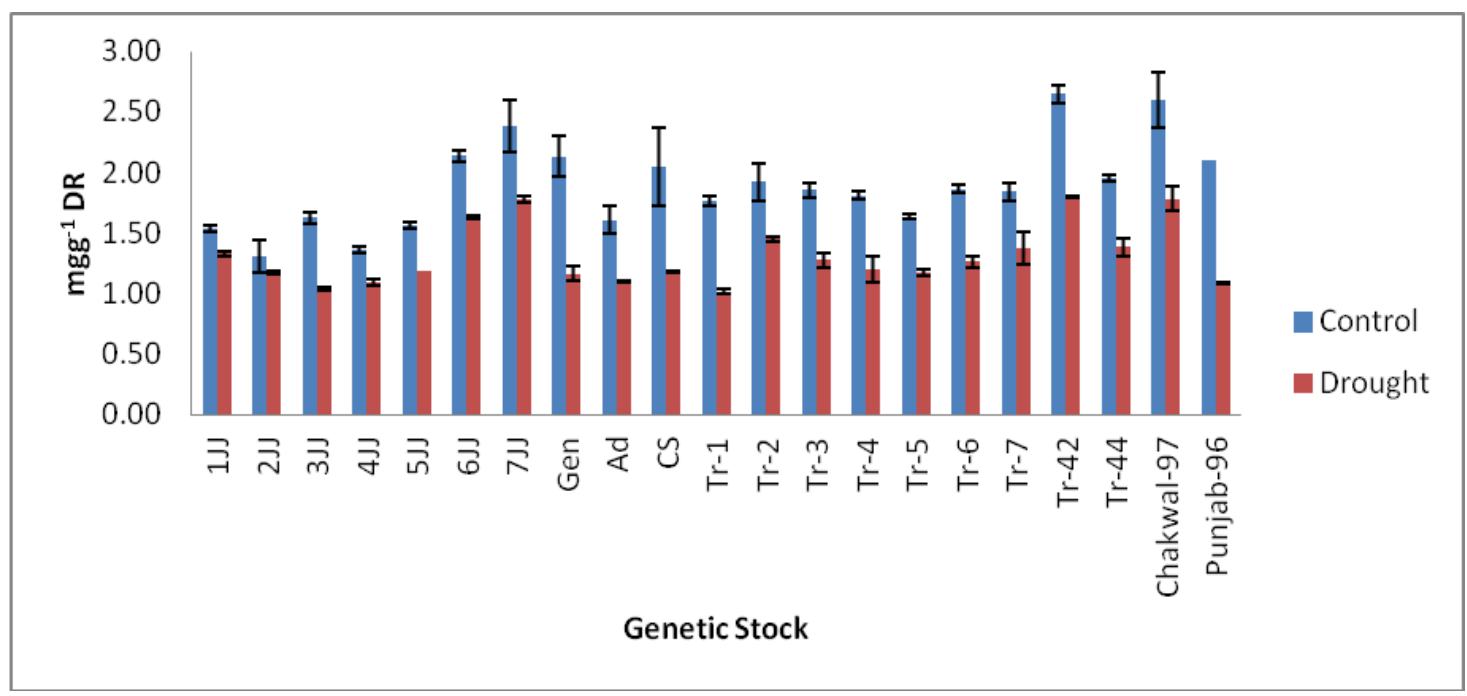

Figure 6. Total chlorophyll contents in leaves at control and 15\% PEG treatments

\section{Discussion}

CS-Th. bessarabicum addition and translocation lines might possess tolerant genes for drought. Salt tolerant species might prove drought tolerant in the areas where water deficit prevails was inferred from the study of Farooq and Azam (2001). Whereas Th. bessarabicum $(2 \mathrm{n}=2 \mathrm{x}=14$, JJ or EbEb) is a perennial maritime wheatgrass that possesses salinity tolerances and can be an important gene source for wheat improvement (Gorham et al., 1985; William and Mujeeb-Kazi, 1993; King et al., 1997a). Majority of the traits associated with improved performance under drought stress have complex nature and controlled by several genes each having a small effect (Rebetzke et al., 2007; Yang et al., 2007).

Drought causes oxidative damages by producing reactive oxygen species (ROS) such as hydroxyls $(\mathrm{OH})$, superoxide $\left(\mathrm{O}_{2}^{-}\right)$, peroxide hydrogen $\left(\mathrm{H}_{2} \mathrm{O}_{2}\right)$. These ROS may initiate disturbance in lipid peroxidation, chlorophyll, protein oxidation, and nucleic acid (Scandalios, 1993). To combat the effects of drought-induced oxidative stress, plants develop complex mechanisms of antioxidant system via induction of drought tolerant genes (Bowler et al., 1992). Increase in ROS causes the enhanced SOD activity which is a major scavenger of $\mathrm{O}_{2}{ }^{-}$and it can catalytic the disproportionate reaction of superoxide radical anions into oxygen and hydrogen peroxide (Scandalios, 1993). As a consequence, $\mathrm{H}_{2} \mathrm{O}_{2}$ accumulation was possibly due to the activation of SOD. Thus, the balance between ROS and the antioxidative system is crucial for survival and adaptation to stress (Weng et al., 2015). Amphiploid, addition lines 1JJ 2JJ, 5JJ, 7JJ and translocation lines $\operatorname{Tr}-2$, Tr-7 might have tried to maintain oxidative stress by exhibiting maximum SOD production. These addition lines possess $1 \mathrm{~J}, 2 \mathrm{~J}, 5 \mathrm{~J}$ and $7 \mathrm{~J}$ chromosomes pairs, while translocation lines have translocated segments of $1 \mathrm{~J}$ and $5 \mathrm{~J}$ from Thinopyrum bessarabicum chromosomes, respectively probably possess genes conferring resistance to drought via overproduction of SOD to scavenge ROS to protect against drought stress. SOD genes were mapped to the long arms of the homologous group-2 and group-7 chromosomes of wheat, respectively (Guohai et al., 1999). Zhao Xinhua et al. (2005) also found QTLs for SOD on chromosomes 1D, 2B and 5A of wheat. Higher SOD activity has also been reported in drought tolerant genotypes of 
wheat (Sariam and Saxena, 2000). Din et al. (2009) also reported enhanced SOD activity in drought tolerant germplasm of wheat.

Drought stress influences the transcription of some of the genes to synthesize different types of drought stress related proteins, and proline is one of them. Proline is a crucial protein that has a vital function in water stress tolerance. Free proline might be involved in membrane stabilization during water stress (Kocheva and Georgiev, 2003), or it might be a reserve of readily mobilizable $\mathrm{N}$ available upon relief of stress (Hare et al., 1998). It has been reported that proline accumulation in the cytosol might be enough for osmotic adjustment by activating other mechanisms, such as the formations of strong H-bonded water around protein for protecting protein structures and scavenger of free radicals (Rascio et al., 1994; Alia et al., 2001; Ghoulam et al., 2002).

Results showed that addition lines 3JJ, 7JJ, 5JJ and translocation lines $\mathrm{Tr}-5$, Tr-6 Tr7 exhibited the highest proline accumulation at $15 \%$ PEG treatment indicating that these addition and translocation lines might possess genes on Th. bessarabicum chromosomes/chromosomal arms, which were induced during water deficit condition to maintain their turgor through osmotic adjustment. Research studies have shown that drought stresses increased leaf proline contents as compared to the control in drought tolerant lines (Dehnavi et al., 2017; Larkunthod et al., 2018). It was indicated by Gariba et al. (1992) that chromosome 4B, 5A and 5D of wheat may carry genes associated with the control of proline accumulation induced by osmotic stress. Ma et al. (2008) indicated an enzyme P5CR is the last enzyme in the cascade for proline synthesis. The TaP5CR gene was located on chromosome 3D of wheat genome. Its expression was upregulated by salt, PEG, ABA and heat stress. On the basis of above mentioned observation it can be suggested that enhanced level of proline accumulation in 5JJ and $3 \mathrm{JJ}$ addition line might be due to extra dosage of genes associated with the control of proline accumulation present on 5J and 3J chromosomes of Th. bessarabicum. The 7JJ addition lines might possess some other genes on Th. bessarabicum chromosomes which might be involved directly or indirectly in enhancing proline accumulation under drought stress. While translocation line Tr-5 contains chromosomal segment of 4J, Tr-6, while Tr-7 contains 6JS and 5JS chromosomal arm of Th. Bessarabicum, respectively that might possess some more genes responsible for inducing stress tolerant genes and accumulation of the highest level of proline under drought condition. Accumulation of osmolyte like proline has also been noticed in variety of crop species. Transgenic tobacco plants over expressing proline synthesis genes have been claimed to induce osmotic adjustment and have increased drought tolerance (Kishore et al., 1995). Under stress condition, proline concentration has been shown to be generally higher in stresstolerant than that of stress-sensitive plants in many plant species such as rice (Shereen et al., 2007), alfalfa (Petrusa and Winicov, 1997), maize (Sharp et al., 1994), pigeon pea (Waheed et al., 2006) and potato (Rahnama and Ebrahimzadeh, 2004).

Concentration of chlorophyll contents in chloroplast affects photosynthetic capacity of crops and thereby considered a significant adaptation trait under water deficit condition (Chernyad'ev and Monakhova, 2003). Tolerance to drought comprises of maintaining high chlorophyll contents under water deficit to support photosynthetic capacity of plants (Yasmin et al., 2013). Drought stress causes decrease in the contents of chlorophyll by producing ROS such as $\mathrm{O}_{2}$ and $\mathrm{H}_{2} \mathrm{O}_{2}$, thereby causing lipid peroxidation which consequently results in chlorophyll destruction (Mirnoff, 1993; Foyer et al., 1994). It has been expressed that tolerant genotypes of wheat and corn possessed higher chlorophyll contents than that of 
sensitive genotypes during oxidative stress (Pastori and Trippi, 1992; Dehnavi et al., 2017). Disomic addition lines 1JJ, 2JJ, 4JJ possess a pair of 1J, 2J, 4J chromosomes and translocation lines $\operatorname{Tr}-7, \operatorname{Tr}-6, \operatorname{Tr}-3, \mathrm{Tr}-4$ possess chromosomal arms 5JS, 6JS, 3JS and $1 \mathrm{JL}$, respectively from $T h$. bessarabicum, emphasizing the importance of these chromosomes/chromosomal arms in determining the tolerance of these genetic stock as these genetic stocks showed minimum reduction in chlorophyll A contents. While 5JJ addition line exhibited an increase in chlorophyll A contents under drought stress might possess gene(s) which were directly or indirectly involved in over expression of the chlorophyll A synthesis genes. It has also been found that the chlorophyll contents of leaves are increased in resistant cultivars under drought stress (Shahriari, 1999).

Reduction in chlorophyll contents of various crops has been previously reported by many researchers under drought stress. Results of chlorophyll B showed that addition lines $1 \mathrm{JJ}, 4 \mathrm{JJ}, 7 \mathrm{JJ}$ and translocation lines $\mathrm{Tr}-1$ and $\mathrm{Tr}-9$ exhibited minimum reduction in chlorophyll $\mathrm{B}$, while addition lines $1 \mathrm{JJ}, 2 \mathrm{JJ}, 4 \mathrm{JJ}, 5 \mathrm{JJ}, 6 \mathrm{JJ}, 7 \mathrm{JJ}$, amphiploid and translocation lines $\operatorname{Tr}-5$ and $\operatorname{Tr}-7$ depicted minimum decrease in total chlorophyll contents under water stress environment. Minimum reduction of chlorophyll B and total chlorophyll contents in these addition and translocation lines under drought stress might be linked to the presence of chromosomes/chromosomal arms from Th. bessarabicum.

It has been demonstrated that chlorophyll concentration in plants is a quantitative trait. Seven QTLs for chlorophyll contents on chromosomes 2B, 4A, 5B, 6A, 7A, and 7D under Nitrogen sufficient environment, while nine QTLs for chlorophyll contents on chromosomes 2D, 3A, 4B, 5B, and 6A when wheat seedlings are grown under nitrogen deficient environment were identified (Cao et al., 2004). Yang et al. (2007) mapped four additive QTLs controlling chlorophyll contents under water deficient and well watered on chromosomes 1A, 5A, and 7A in wheat. All these observations specify that controlling of chlorophyll contents in various environments is governed by multiple gene loci distributed on different chromosomes authenticating it as quantitative trait. Similar kinds of increased stress tolerance through presence of alien chromosomes of Th. Elongatum have also been reported by Taeb et al. (1993). Several alien substitution and translocation lines of wheat (Triticum aestivum L.) have shown genetic diversity for desirable traits of the alien donors (Rabinovich, 1998; Ko et al., 2002).

\section{Conclusion}

Wheat-Th. bessarabicum genetic stocks were given 15\% PEG stress to evaluate their drought tolerance. The results indicated that amphiploid and the addition lines $1 \mathrm{JJ}, 5 \mathrm{JJ}$, and translocation line $\mathrm{Tr}-7$ have performed best in most of the physiological parameters studied in drought stress, and are considered to be tolerant under water stress condition. Other addition lines including $2 \mathrm{JJ}, 4 \mathrm{JJ}$ and $7 \mathrm{JJ}$ can be categorized as moderately tolerant to water deficit condition on the basis of their performance in traits studied under drought stress. These addition and translocation lines are recommended as sources of introducing drought tolerance into drought susceptible wheat cultivars.

Acknowledgements. This work is a part of Ph.D. Dissertation and supported by the Hazara University and National Agriculture Research Centre (NARC), Pakistan. 


\section{REFERENCES}

[1] Alia, P. M., Matysik, J. (2001): Effect of proline on the production of singlet oxygen. Amino Acids 21: 195-200.

[2] Araus, J. L., Bort, J., Steduto, P., Villegas, D., Royo, C. (2003): Breeding cereals for Mediterranean conditions: ecophysiological clues for biotechnology application. - Annals of Applied Biology 142: 129-141.

[3] Arnon, D. I. (1949): Copper enzymes in isolated chloroplast polyphenol oxidase in Beta vulgaris. - Plant Physiology 24: 1-15.

[4] Ashraf, M., Ozturk, M., Athar, H. R. (2009): Salinity and Water Stress. Improving Crop Efficiency. - Springer-Verlag, Berlin.

[5] Bano, A., Ullah, F., Nousheen, A. (2012): Role of abscisic acid and drought stress on the activities of antioxidant enzymes in wheat. - Plant Soil Environment 58: 181-185.

[6] Bates, L. S., Waldren, R. P., Teare, L. D. (1973): Rapid determination of free proline for water stress studies. - Plant Soil 39: 205-207.

[7] Blum, A. (1998): Improving wheat grain filling under stress by stem reserve mobilization. - Wheat: Prospects for Global Improvement 100: 77-83.

[8] Bowler, C., Montagu, V., Inze, M. D. (1992): Superoxide dismutase and stress tolerance. - Annual Review of Plant Physiology and Plant Molecular Biology 43: 83-116.

[9] Bray, E. A. (1993): Molecular responses to water deficit. - Plant Physiology 103: 10351040.

[10] Cao, W. D., Jia, J. Z., Jin, J. Y. (2004): Identification and interaction analysis of QTL for chlorophyll content in wheat seedlings. - Plant Nutrition and Fertilizer Science 10: 473478.

[11] Chernyadev, I. I., Monakhova, O. F. (2003): Effects of cytokinin preparations on the pools of pigments and proteins of wheat cultivars differing in their tolerance to water stress. - Applied Biochemistry and Microbiology 39: 524-531.

[12] Din, J., Khan, S. U., Ali, I. (2009): Physiological assessment of drought tolerance in wheat (Triticum aestivum L.) varieties under moisture stress conditions. - Biologia Pakistan 55: 1-9.

[13] Dehnavi, M. M., Zare, T., Khajeeyan, R., Merajipoor, M. (2017): Drought and salinity impacts on bread wheat in a hydroponic culture: a physiological comparison. - Journal of Plant Physiology and Breeding 7: 61-74.

[14] Farooq, S., Azam, F. (2001): Co-existence of salt and drought tolerance in Triticeae. Hereditas 135: 205-210.

[15] Foyer, C. H., Descourvieres, P., Kunert, K. J. (1994): Photo oxidative stress in plants. Plant Physiology 92: 696-717.

[16] Galiba, G., Simon-Sarkadi, L., Kocsy, G., Salgo, A., Sutka, J. (1992): Possible chromosomal location of genes determining the osmoregulation of wheat. - Theoretical Applied Genetics 85: 415-418.

[17] Ghoulam, C. H., Foursy, A., Fares, K. (2002): Effects of salt stress on growth, inorganic ions and proline accumulation in relation to osmotic adjustment in five sugar beet cultivars. - Environmental and Experimental Botany 47: 39-50.

[18] Giannopolitis, C. N., Ries, S. K. (1977): Superoxide dismutases: I. Occurance in higher plants. - Plant Physiology 59: 309-314.

[19] Gorham, J., McDonnell, E., Budrewicz, E., Wynn Jones, R. G. (1985): Salt tolerance in the Triticeae: growth and solute accumulation in leaves of Thinopyrum bessarabicum. Journal of Experimental Botany 36: 1021-1031.

[20] Guohai, W., Ronald Wilen, W., Robertson, J. A., Gusta, L. V. (1999): Isolation, chromosomal localization, and differential expression of mitochondrial manganese superoxide dismutase and chloroplastic copper/zinc superoxide dismutase genes in heat. Plant Physiology 120: 513-520. 
[21] Hare, P. D., Cress, W. A., Staden, J. (1998): Dissecting the role of osmolyte accumulation during stress. - Plant Cell and Environment 21: 535-553.

[22] Hoffmann, W. A., Marchin, R. M., Abit, P., Lau, O. L. (2011): Hydraulic failure and tree dieback are associated with high wood density in a temperate forest under extreme drought. - Global Change and Biology 17: 2731-2742.

[23] Jones, H. G., Corlett, J. E. (1992): Current topics in drought physiology. - Journal of Agricultural Science 119: 291-296.

[24] King, I. P., Forster, B. P., Law, C. C., Cant, K. A., Orford, S. E., Gorham, J., Reader, S., Miller, T. E. (1997a): Introgression of salt-tolerance genes from Thinopyrum bessarabicum into wheat. - New Phytology 137: 75-81.

[25] Kishore, K. P. B., Hong, Z., Miao, G. H., Hu, C. A. A., Verma, D. P. S. (1995): Over expression of Delta (1)-pyrroline-5-carboxylate synthetase increases proline production and confers osmotolerance in transgenic plants. - Plant Physiology 108: 1387-1394.

[26] Ko, J. M., Seo, B. B., Suh, D. Y., Do, G. S., Park, D. S., Kwack, Y. H. (2002): Production of a new wheat line possessing the 1BL.1RS wheat-rye translocation derived from Korean rye cultivar Paldanghomil. - Theoretical Applied Genetics 104: 171-176.

[27] Kocheva, K., Georgiev, G. (2003): Evaluation of the reaction of two contrasting barley (Hordeum vulgare L.) cultivars in response to osmotic stress with PEG 6000. - Belgium Journal of Plant Physiology 2003(Special Issue): 290-294.

[28] Larkunthod, P., Nounjan, N., Siangliw, J. L., Toojinda, T., Sanitchon, J., Jongdee, B., Theerakulpisut, P. (2018): Physiological responses under drought stress of improved drought-tolerant rice lines and their parents. - Notulae Botanicae Horti Agrobotanici Cluj-Napoca 46: 679-687.

[29] Lonbani, M., Arzani, A. (2011): Morpho-physiological traits associated with terminal drought stress tolerance in triticale and wheat. - Agronomy Research 9: 315-329.

[30] Ma, L., Zhou, E., Gao, L., Mao, X., Zhou, R., Jia, J. (2008): Isolation, expression analysis and chromosomal location of P5CR gene in common wheat (Triticum aestivum L.) S. African Journal of Botany 74: 705-712.

[31] Majid, S. A., Asghar, R., Murtaza, G. (2007): Yield stability analysis conferring adaptation of wheat to pre-and post-anthesis drought conditions. - Pakistan Journal of Botany 39: 1623-1637.

[32] Martin, M., Micell, F., Morgan, J. A., Scalet, M., Zerbi, G. (1993): Synthesis of osmotically active substances in winter wheat leaves as related to drought resistance of different genotypes. - Journal of Agronomy and Crop Science 171: 176-184.

[33] Mirnoff, N. (1993): The role of active oxygen in the response of plants to water deficit and desiccation. - New Phytology 125: 27-58.

[34] Mittler, R. (2006): Abiotic stress, the field environment and stress combination. - Trends in Plant Science 11: 15-19.

[35] Morris, M. L., Blaid, A., Byerlee (1991): Wheat and Barley Production in Rain Fed Marginal Environments of the Developing World. Part I of 1990-91. - CIMMYT, Mexico.

[36] Mujtaba, S. M., Alam, S. M. (2002, April): Drought phenomenon and crop growth. Industry \& Economy. Retrieved http://www.pakistaneconomist.com/issue2002/issue13/i\&e4.htm

[37] Nayyar, H., Walia, D. P. (2003): Water stress induced proline accumulation in contrasting wheat genotypes as affected by calcium and abscisic acid. - Biology of Plant 46: 275279.

[38] Nevo, E. (2004): Genomic Diversity in Nature and Domestication. - In: Henry, R. (ed.) Diversity and Evolution of Plants. Genotypic and Phenotypic Variation in Higher Plants. CAB International, Wallingford, UK, pp. 287-316.

[39] Passioura, J. B. (2007): The drought environment: physical, biological and agricultural perspectives. - Journal of Experimental Botany 58: 113-117. 
[40] Pastori, G. M., Trippi, V. S. (1992): Oxidative stress induces high rate of glutathione reductase synthesis in a drought-resistant maize strain. - Plant Cell Physiology 33: 957961.

[41] Pessarkli, M. (1999): Handbook of Plant and Crop Stress. - Marcel Dekker Inc., New York.

[42] Petrusa, L. M., Winicov, I. (1997): Proline status in salt tolerant and salt sensitive alfalfa cell lines and plants in response to NaCl. - Plant Physiology and Biochemistry 35: 303310.

[43] Rabinovich, S. V. (1998): Importance of wheat-rye translocations for breeding modern cultivars of Triticum aestivum L. - Euphytica 100: 323-340.

[44] Rahnama, H., Ebrahimzadeh, H. (2004): The effect of $\mathrm{NaCl}$ on proline accumulation in potato seedlings and calli. - Acta Physiology of Plant 26: 263-270.

[45] Rascio, A., Plantani, C., Sealfati, G., Tonti, A., Di Fonzo, N. (1994): The accumulation of solutes and water binding strength in durum wheat. - Physiology of Plant 90: 554-558.

[46] Rebetzke. G. J., Ellis, M. H., Bonnett, D. G., Richards, R. A. (2007): Molecular mapping of genes for coleoptile growth in bread wheat (Triticum aestivum L.). - Theoretical Applied Genetics 114: 1173-1183.

[47] Robin, A. H. K., Uddin, M. J., Bayazid, K. N. (2015): Polyethylene glycol (PEG)-treated hydroponic culture reduces length and diameter of root hairs of wheat varieties. Agronomy 5: 506-518.

[48] Sairam, R. K., Saxena, D. C. (2000): Oxidative stress and antioxidants in wheat genotypes: possible mechanism of water stress tolerance. - Journal of Agronomy and Crop Science 184: 55-61.

[49] Scandalios, J. G. (1993): Oxygen stress and superoxide dismutases. - Plant Physiology 101: 7-12.

[50] Sharp, R. E., Wu, Y., Voetberg, G. S., Saab, I. N., LeNoble, M. E. (1994): Confirmation that abscisic acid accumulation is required for maize primary root elongation at low water potentials. - Journal of Experimental Botany 45: 1717-1743.

[51] Shereen, A., Ansari, R. U., Yasmin, S., Raza, S., Mumtaz, S., Khan, M. A., Mujtaba, S. M. (2007): Physiological response of rice (Oryza sativa L.) to saline stress. - Pakistan Journal of Botany 39: 2527-2534.

[52] Taeb, M., Koebner, R. M. D., Forster, B. P. (1993): Genetic variation for water logging tolerance in the Triticeae and the chromosomal location of genes conferring water logging tolerance in Thinopyrum elongatum. - Genome 36: 825-830.

[53] Waheed, A., Hafiz, I. A., Qadir, G., Murtaza, G., Mahmood, T., Ashraf, M. (2006): Effect of salinity on germination, growth, yield, ionic balance and solute composition of pigeon pea (Cajanus cajan (L.) Mill sp). - Pakistan Journal of Botany 38: 1103-1117.

[54] Weng, M., Cui, I., Liu, F., Min, Z., Shan, I., Yang, S., Deng, X. (2015): Effects of drought stress on antioxidant enzymes in seedlings of different wheat genotypes. Pakistan Journal of Botany 47: 49-56.

[55] William, M. D. H., Mujeeb-Kazi, A. (1993): Thinopyrum bessarabicum: Biochemical and cytological markers for the detection of genetic introgression in its hybrid derivatives with Triticum aestivum L. - Theoretical Applied Genetics 86: 365-370.

[56] Xie, W., Nevo, E. (2008): Wild emmer: Genetic resources, gene mapping and transfer for wheat improvement. - Euphytica 164: 603-614.

[57] Yang, D. L., Jing, R. L., Chang, X. P., W, Li. (2007): Identification of quantitative trait loci and environmental interactions for accumulation and remobilization of water-soluble carbohydrates in wheat (Triticum aestivum L.) stems. - Genetics 176: 571-584.

[58] Yasmeen, A., Basra, S. M. A., Wahid, A., Farooq, M., Nouman, W., Rehman, H. U., Hussain, N. (2013): Improving drought resistance in wheat (Triticum aestivum) by exogenous application of growth enhancers. - International Journal of Agriculture and Biology 15: 1307-1312. 
[59] Zhao, L. Y., Deng, X. P., Shan, L. (2005): Effects of osmotic stress on chlorophyll fluorescence parameters of wheat seedling. - Chinese Journal of Applied Ecology16: 1261-1264.

[60] Zu, X., Lu, Y., Wang, Q., Chu, P., Miao, W., Wang, H., La, H. (2017): A new method for evaluating the drought tolerance of upland rice cultivars. - The Crop Journal 5: 488-498. 